\title{
Comparison of the Effectiveness of Two Types of Single Port Minimal Invasive Neurosurgical Robots to Ablation and Resection of Brain Tumor
}

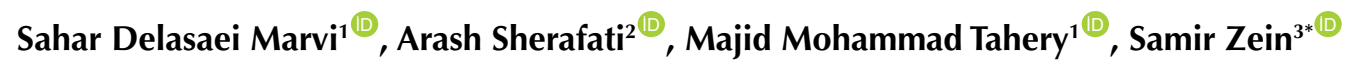 \\ ${ }^{1}$ Department of Biomedical Engineering, North Tehran Branch, Islamic Azad University, Tehran, Iran. \\ ${ }^{2}$ Computer Engineering Department, Shomal University, Amol, Mazandaran, Iran. \\ ${ }^{3}$ Department of Biomedical Engineering, Science and Research Branch, Islamic Azad University, Tehran, Iran.
}

\begin{abstract}
Background:Using minimally invasive neurosurgical robots is one of the most desirable ablation methods and resection of brain tumors. In this study, forward kinematics and Jacobian matrix calculated for two single-port robots for comparing the effectiveness of two types of single port minimal invasive surgical robots to ablation and resection of brain tumor

Methods: The motion analysis of robots type 1 and 2 has compared to each other. Ablation manipulator in robot type 1 has five degrees of freedom, but in robot type 2, three revolute degrees of freedom of this manipulator has replaced with a revolute joint perpendicular to the previous three revolute joints.

Results: Results showed that for resection surgery, in the same conditions, robot type 2 damaged $58.9 \mathrm{~mm}^{3}$ more of cerebral cortex tissue than robot type 1 to resect the brain tumors. To establish a static balance, robot type 2 needs to tolerate at least $41 \%$ more internal loading than robot type 1. The maximum velocity for robot type 1 in the contact location between the end-effector and the tumor is 1.7 times more than robot type 2. The maximum end-effector force of robot type 1 to apply the tumor for ablation surgery is more than 1.8 times in robot type 2 , but the maximum moment and power for ablation surgery and resection of these two robots were the same less than $1 \%$ difference. Conclusion: Despite the more straightforward mechanism, a minimum number of joints, and better kinematics range of robot type 2, robot types 1 has the possibility for transformation, establishes the static balancing, and does a better ablation surgery with less damage to the brain.

Keywords: Surgical robot; Ablation of the tumor; Force; resection of tumor; Brain tumor.
\end{abstract}

*Correspondence to

Samir Zein, Department of

Biomedical Engineering,

Science and Research Branch, Islamic Azad University, Tehran, Iran.

Tel-Fax: (+98-21) 44865154

Email: Samir.zein@srbiau.ac.ir

Published online 28 September 2020

Citation: Delasaei Marvi S, Sherafati A, Tahery MM, Zein S. Comparison of the Effectiveness of Two Types of Single Port Minimal Invasive Neurosurgical Robots to Ablation and Resection of Brain Tumor Clin Neurosci J. 2020;7(4):201-207. doi:10.34172/icnj.2020.26.

\section{Introduction}

The first practical use of robotics in medical science dates back to $1985^{1}$. Following these systems' improvement, robotics is used in several fields such as neurosurgery, ${ }^{2-9}$ cell biology, ${ }^{10,11}$ rehabilitation, ${ }^{12}$ orthopedic surgeries, ${ }^{13,}{ }^{14}$ laparoscopies, ${ }^{15}$ and cardiovascular surgeries. ${ }^{16-18}$ In recent years, minimally invasive surgery methods have attracted much attention. In robotic surgery, the system has divided into two parts: master device, controlled by the surgeon or the surgery operator, and the commands from this part are transferred to the second part, slave. The slave has consisted of robot arms that execute the surgery. One of the most popular systems is the da Vinci surgical robot, and one of its common models is multiport. This robot has three arms. Two of them hold the surgery parts, and the remaining one holds the endoscope. ${ }^{11} 19$ The main problem of using multiport robots, especially in surgeries with deep target points, is the need for creating more piercing. Furthermore, there is a risk of collision of the robot arms with each other. ${ }^{20}$ A tool named VeSPA has designed for da Vinci robot, which has a multichannel port and is useful, especially in urology surgeries. ${ }^{14}$

One of the most common neurosurgeries is about the resection of tumors from the brain cortex, and it has performed in two ways. The first method is open surgery. In this method, a large amount of the skull opened, and the lesion directly removed from the brain. The second method is using surgical robots. In this method, a limited part of the brain in which the tumor exists has specified using the imaging method, image guide, and virtual reality. Then through a hole as small as possible, the tumor is removed from the brain. ${ }^{21,22}$ One advantage of 
the second method is the small surgical site's small size, which results in less pain for the patient after the surgery, shorter recovery duration, and, therefore, fewer hospital costs. ${ }^{1,20}$

$\mathrm{Hu}$ et al introduced a semi-autonomous surgical robot that can resection of a brain tumor. ${ }^{23}$ It should note that this robot has stereo visual feedback too. They used two plans to resection the tumor and compared the autonomous motion control method and visual endeffector sensing for each plan. Chinbe et al. designed a finger-attachment manipulator system for a resection brain tumor which operates based on pulling force feedback ${ }^{24}$. Their focus was on robots with more accuracy and safe resection. Azarnoush et al. used a special manipulator for brain tumor resection in which safety has improved during resection by utilizing modulating resident force application ${ }^{25}$. Niccolini et al. proposed a platform for neuro-endoscopy, which results in more accuracy of robots both in autonomous and hands-on control methods by using an accurate tool ${ }^{26}$. Lefranc et al. also introduced a frameless robot for posterior fossa biopsy ${ }^{27}$. This proposed platform results in more accuracy and safety in comparison with normal biopsies in neurosurgery. Maddahi et al. built a robot-assisted micro-neurosurgery, which creates the optimal position, orientation, and force using a hand-controller in the robot workspace area ${ }^{28}$. Ross et al. designed a manipulator with two axes that operated the tumor resection by a cutting laser ${ }^{29}$. They used a feed-forward to provide automated soft tissue resection in these robots.

Despite sensitivities in neurosurgery and several limitations in robot workspace, improving robots' work efficiency by optimizing their joints is not studied before. Therefore, the present study attempts to answer this need in single-port minimal invasive surgery for brain tumor removal while preserving the accuracy and design considerations. This goal aims two types of single-port minimal invasive surgery robots have considered, and their performance has compared.

\section{Materials and Methods}

According to the present study's purpose, a single-port minimal invasive surgery robot is named robot type 1 and has compared with the developed model of this robot named robot type 2 . Robot type 1 has tubular housing in its arm and end-effector, containing two small manipulators (Figure 1). This tubular housing has a $20 \mathrm{~mm}$ diameter and enables a robot for translation movement along the horizontal axis. After skull drilling (Figure 1a) and placing a trocar, this tubular housing had placed to make a tumor pathway. It should note that the trocar diameter and endoscope of this robot are 20.2 $\mathrm{mm}$ and $10 \mathrm{~mm}$, respectively. This robot consists of two manipulators (Figure 1b). The first manipulator has 5 degrees of freedom and allows for resection and ablation of tumors from the brain cortex (Figure 2 -Zoom A and B). These 5 degrees of freedom consist of 4 revolute degrees of freedom and one prismatic degree of freedom. Revolute degree of freedom is for end-effector proper access to all the tumor parts (Figure 2 -Zoom B). One prismatic degree of freedom had used for covering all the tumor volumes for accessing the tumor's depth in the brain cortex. The second manipulator is called suction and has 4 degrees of freedom. This manipulator sucks the separated tumor parts and removes them from the brain. This manipulator has three revolute degrees of freedom for accessing all the resected tumor volume and one prismatic degree of freedom to remove a tumor from the skull. The diameter of these manipulators is $4 \mathrm{~mm}$. It should note that the ablation manipulator has one more revolute degree of freedom than a suction manipulator, which is because of the installed gripper in the endeffector of this manipulator.

In robot type 2 , at the junction of the manipulator to the tubular housing, instead of using three revolute joints like in robot type 1 (Figure 3 - Zoom A), 1 is a joint perpendicular to the other three revolute joints used. The important fact is that this new robot's ease of operation or higher efficiency depends on its output and kinematic and kinetic functions, which will discuss in the following (a)

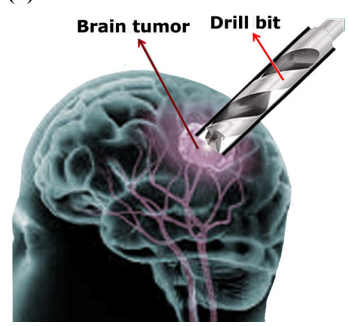

(b)

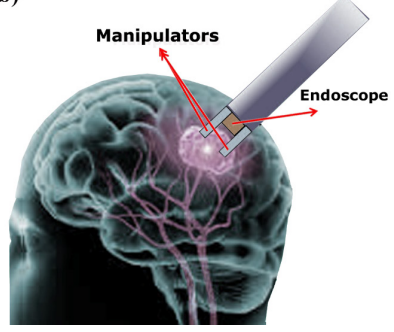

Figure 1. Schematic of brain tumor and single port minimal invasive surgical robots. a) skull drilling and placing a trocar, b) robot component.

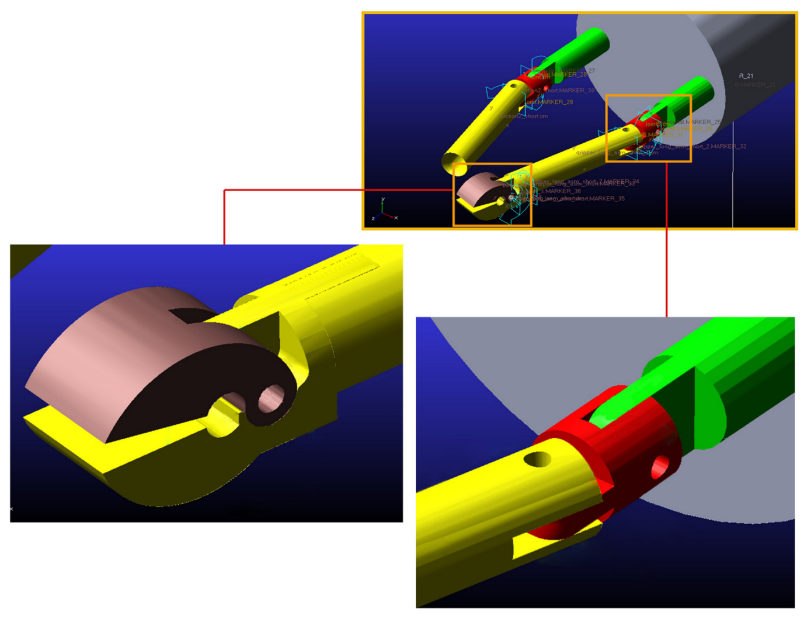

Figure 2. The manipulators of robot type 1 and its components 


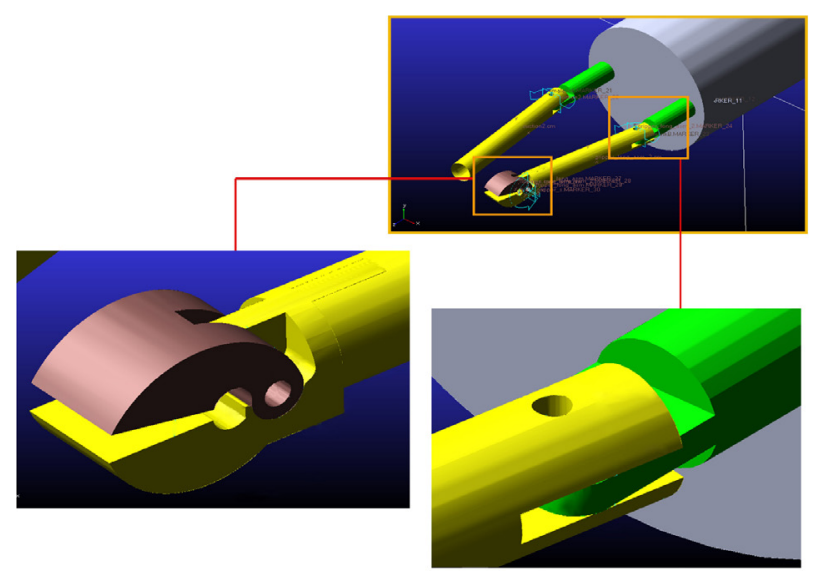

Figure 3. The manipulators of robot type 2 and its components.

sections.

\section{Results}

\section{Transformation and spatial description}

Forward kinematic analysis is one of the most important methods for a robot transformation description. Therefore, for both robot types, the table of DenavitHartenberg (D-H) parameters was calculated (Tables 1 and 2).

The transformation matrix derived from Equation 1 is a compilation of $\mathrm{R}_{3 \times 3}$ and $\mathrm{P}_{3 \times 3}$ matrices ${ }^{22}$, calculated for both robot types using forward kinematics.

${ }_{i}^{i-1} T=\left[\begin{array}{cc}{ }_{i}^{i-1} R & { }_{i}^{i-1} P \\ 0 & 1\end{array}\right\rfloor=\left[\begin{array}{cccc}C \theta_{i} & -S \theta_{i} & 0 & \alpha_{i-1} \\ S \theta_{i} c \alpha_{i-1} & C \theta_{i} c \alpha_{i-1} & -s \alpha_{i-1} & -S \theta_{i} d_{i} \\ S \theta_{i} s \alpha_{i-1} & C \theta_{i} s \alpha_{i-1} & c \alpha_{i-1} & c \alpha_{i-1} d_{i} \\ 0 & 0 & 0 & 1\end{array}\right\rfloor$

Equations 2 and 3 describe the transformation matrix for robots type 1 and 2 , respectively.

The transformation matrix had used for comparing orientation, spatial description, and location of type1 and type 2 robots. By multiplying this matrix with robot end-effector location, initial location, and orientation calculated.

$$
\begin{aligned}
& { }_{5}^{0} T=\left[\begin{array}{cccc}
r_{11} & r_{12} & r_{13} & r_{14} \\
r_{21} & r_{22} & r_{23} & r_{24} \\
r_{31} & r_{32} & r_{33} & r_{34} \\
0 & 0 & 0 & 1
\end{array}\right\rfloor \\
& r_{11}=S \theta_{2} s \\
& \theta_{4}+C \theta_{2} C \theta_{3} C \theta_{4} \\
& r_{12}=C \theta_{4} S \theta_{2}-C \theta_{2} C \theta_{3} S \theta_{4} \\
& r_{13}=S \theta_{2}+C \theta_{2} S \theta_{3}
\end{aligned}
$$

$$
\begin{aligned}
& r_{14}=L_{1}\left(S \theta_{2} S \theta_{4}+C \theta_{2} C \theta_{3} C \theta_{4}\right) \\
& r_{21}=C \theta_{3} C \theta_{4} S \theta_{2}-C \theta_{2} S \theta_{4} \\
& r_{22}=-C \theta_{2} C \theta_{4}-C \theta_{3} S \theta_{2} S \theta_{4} \\
& r_{23}=S \theta_{2} S \theta_{3}-C \theta_{2} \\
& r_{24}=-L_{1}\left(C \theta_{2} S \theta_{4}-C \theta_{3} C \theta_{4} S \theta_{2}\right) \\
& r_{31}=S \theta_{4}+C \theta_{4} S \theta_{3} \\
& r_{32}=C \theta_{4}-S \theta_{3} S \theta_{4} \\
& r_{33}=1-C \theta_{3}
\end{aligned}
$$$$
r_{34}=d_{1}+L_{1}\left(S \theta_{4}+C \theta_{4} S \theta_{3}\right)
$$$$
{ }_{3}^{0} T=\left[\begin{array}{cccc}
C \theta_{2} & 0 & S \theta_{2} & L_{1} C \theta_{2} \\
S \theta_{2} & 0 & -C \theta_{2} & L_{1} S \theta_{2} \\
0 & 1 & 0 & d_{1} \\
0 & 0 & 0 & 1
\end{array}\right\rfloor
$$

In these calculations, $\mathrm{S} \theta$ and $\mathrm{C} \theta$ are $\operatorname{Sin}(\theta)$ and $\operatorname{Cos}(\theta)$, respectively and head center is considered as the origin of coordinates. Thus, tumor location which is the final destination of the robot's end-effector was considered in the same condition for both robot types at position $[35,35,0]^{\mathrm{T}}$ (numbers unit is $\mathrm{mm}$ ). The location of the initial origin for both robot types was compared due to

Table 1. The D-H parameters for ablation manipulator of robot type 1

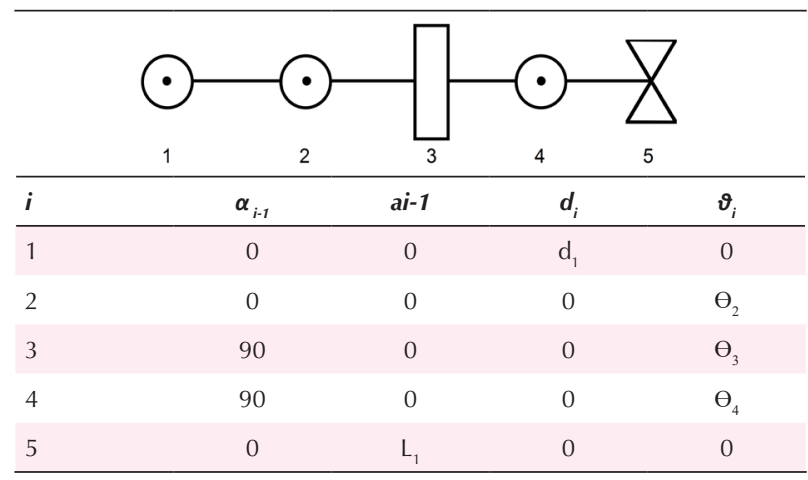

Table 2. The D-H parameters for ablation manipulator of robot type 2

\begin{tabular}{lcccc}
\hline $\boldsymbol{i}$ & $\boldsymbol{\alpha}_{\boldsymbol{i}-\boldsymbol{1}}$ & $\boldsymbol{a}_{\boldsymbol{i}-1}$ & $\boldsymbol{d}_{\boldsymbol{i}}$ & $\boldsymbol{\vartheta}_{\boldsymbol{i}}$ \\
\hline 1 & 0 & 0 & $\mathrm{~d}_{1}$ & 0 \\
2 & 0 & 0 & 0 & $\Theta_{2}$ \\
3 & 90 & $\mathrm{~L}_{1}$ & 0 & 0 \\
\hline
\end{tabular}


equations 4 and 5. For accessing the same location for end-effector of both robot types with $=\left[15^{\circ}, 10^{\circ}, 30^{\circ}, 1 \mathrm{~cm}\right.$, $2 \mathrm{~cm}]^{\mathrm{T}}\left[\theta_{2}, \theta_{3}, \theta_{4}, L_{1}, d_{1}\right]^{\mathrm{T}}$ coordinates, the result is as follows: initial location of robot type 1 and robot type 2 are $\mathrm{x}=14.1, \mathrm{y}=-52.3, \mathrm{z}=-41.6$ and $\mathrm{x}=-34.2, \mathrm{y}=29.3, \mathrm{z}=55.0$ $\mathrm{mm}$ respectively (Equations 4 and 5 ). According to these equations, the distance traveled by robot type 1 and robot type 2 for accessing the same tumor is 68.2 and $71.2 \mathrm{~mm}$, respectively. Therefore, although robot type 2 uses fewer joints and has a simpler mechanism, it has limited ability for spatial movement and transformation in comparison with robot type 1. According to Equation 6, during resection surgery robot type 2 damaged cerebral cortex tissue $58.9 \mathrm{~mm}^{3}$ more than robot type 1 for resection of ablated tumors. Since robot type 2 damages cortex more than robot type 1, using it may increase the chances of post-surgery damages in the patient which can cause a longer recovery process.

${ }_{5}^{0} T=\left[\begin{array}{cccc}-0.2554 & 0.7301 & 1.0636 & -0.2554 \\ -0.8348 & -0.4219 & 0.4059 & -0.8348 \\ -1.0719 & -0.3833 & 1.8391 & 0.9281 \\ 0 & 0 & 0 & 1\end{array}\right\rfloor{ }_{5}^{0} T \times\left\lfloor\left[\begin{array}{c}35 \\ 35 \\ 0 \\ 1\end{array}\right]=\left\lfloor\begin{array}{c}14.1 \\ -52.3 \\ -41.6 \\ 1\end{array}\right\rfloor \mathrm{mm}\right.$

${ }_{3}^{0} T=\left[\begin{array}{cccc}-0.7597 & 0 & 0.6503 & -0.7597 \\ 0.6503 & 0 & 0.7597 & 0.6503 \\ 0 & 1 & 0 & 2 \\ 0 & 0 & 0 & 1\end{array}\right\rfloor,{ }_{3}^{0} T \times\left[\begin{array}{c}35 \\ 35 \\ 0 \\ 1\end{array}\right\rfloor=\left\lfloor\begin{array}{c}-34.2 \\ 29.3 \\ 55.0 \\ 1\end{array}\right\rfloor \mathrm{mm}$

$V_{\text {Damaged }}=\pi r^{2} \times h=\pi(2.5)^{2} \times(71.2-68.2)=58.9 \mathrm{~mm}^{3}$

\section{Jacobian matrix}

The Jacobian determinant had used when making a change of variables when evaluating multiple integrals of a function over a region within its domain. The Jacobian can also solve differential equations at an equilibrium point or approximate solutions near an equilibrium point. For evaluating and comparing manipulators balancing for both robot types, their joints' input needs to be analyzed and compared with each other. To do this, the Jacobian matrix for both robot types calculated. This matrix has six rows, and the number of its columns equals the number of that robot joints. The first three rows (Jv) derived from matrix $\mathrm{P}_{3 \times 1}$ relative to joints variables $(\mathrm{q})$ and the second 3 rows derived from matrix $\mathrm{R}$ of each joint relative to another joint. Equations 7 and 8, show a Jacobian matrix for robot type 1 and type 2 , respectively.

$$
J_{0-5}=\left[\begin{array}{ccccc}
0 & J_{12} & J_{13} & J_{14} & 0 \\
0 & J_{22} & J_{23} & J_{24} & 0 \\
1 & 0 & J_{33} & J_{34} & 0 \\
0 & 0 & J_{43} & J_{44} & J_{45} \\
0 & 0 & J_{53} & J_{54} & J_{55} \\
0 & 1 & 1 & J_{64} & J_{65}
\end{array}\right]
$$

$$
\begin{aligned}
& J_{12}=L_{1}\left(C \theta_{2} S \theta_{4}-C \theta_{3} C \theta_{4} S \theta_{2}\right) \\
& J_{22}=L_{1}\left(S \theta_{2} S \theta_{4}+C \theta_{2} C \theta_{3} C \theta_{4}\right) \\
& J_{13}=-L_{1} C \theta_{2} C \theta_{4} S \theta_{3} \\
& J_{23}=-L_{1} C \theta_{4} S \theta_{2} S \theta_{3} \\
& J_{33}=L_{1} C \theta_{3} C \theta_{4} \\
& J_{43}=S \theta_{2} \\
& J_{53}=-C \theta_{2} \\
& J_{14}=L_{1}\left(C \theta_{4} S \theta_{2}-C \theta_{2} C \theta_{3} S \theta_{4}\right) \\
& J_{24}=-L_{1}\left(C \theta_{2} C \theta_{4}+C \theta_{3} S \theta_{4} S \theta_{2}\right) \\
& J_{34}=L_{1}\left(C \theta_{4}-S \theta_{4} S \theta_{3}\right) \\
& J_{44}=S \theta_{2}+C \theta_{2} S \theta_{3} \\
& J_{54}=S \theta_{2} S \theta_{3}-C \theta_{2} \\
& J_{64}=1-C \theta_{3} \\
& J_{45}=S \theta_{2}+C \theta_{2} S \theta_{3} \\
& J_{55}=S \theta_{2} S \theta_{3}-C \theta_{2} \\
& J_{65}=1-C \theta_{3} \\
& J_{0-3}=\left[\begin{array}{ccc}
0 & -L_{1} S \theta_{2} & 0 \\
0 & L_{1} C \theta_{2} & 0 \\
1 & 0 & 0 \\
0 & 0 & S \theta_{2} \\
0 & 0 & -C \theta_{2} \\
0 & 1 & 0
\end{array}\right]
\end{aligned}
$$

Robot's external loading, which is the tumor weight, should be applied in both robot types. According to a normal and minimum weight for a brain tumor, this number for each robot type considered being $0.5 \mathrm{~N}$, so that robots have compared in the same condition. Thus, both robots' external loading matrix has considered being $[0,-0.5 \mathrm{~N}, 0]^{\mathrm{T}}$. Equations 9 and 10 are calculated from multiplying transpose of the Jacobian matrix of robot type 1 and robot type 2 with an external loading matrix. The results showed that joints input matrix for robot type 1 and type 2 for establishing static balancing is [0, 0.27, $0,0.21,0]^{\mathrm{T}}$ and $[0,0.38,0]^{\mathrm{T}}$, respectively. This means that for establishing static balancing in robot type 1 , the second and fourth joints should engage, and in the same 
condition, the second joint should be engaged for robot type 2 . However, robot type 2 needs to endure 41 percent more internal loading than robot type 1 to supply static balancing, which results in more depreciation of the robot. If this robot's vibration conditions have not designed well, it can cause several errors during surgery. While surgeries in the neuroscience field are very sensitive, these small errors may cause irreparable damages to the patient.

$J_{0-5}=\left[\begin{array}{ccccc}0 & 0.8348 & -0.0638 & 0.7301 & 0 \\ 0 & -0.5442 & 0.0546 & -0.4219 & 0 \\ 1 & 0 & -0.1294 & -0.3833 & 0 \\ 0 & 0 & 0.6503 & 1.0636 & 1.0636 \\ 0 & 0 & 0.7597 & 0.4059 & 0.4059 \\ 0 & 1 & 1 & 1.8391 & 1.8391\end{array}\right], F_{5}=J_{0-5}^{T} \times\left[\begin{array}{c}0 \\ -0.5 \\ 0 \\ 0 \\ 0 \\ 0\end{array}\right]=\left[\begin{array}{c}0 \\ 0.27 \\ 0 \\ 0.21 \\ 0\end{array}\right]$

$$
J_{0-3}=\left[\begin{array}{ccc}
0 & -0.6503 & 0 \\
0 & -0.7597 & 0 \\
1 & 0 & 0 \\
0 & 0 & 0.6503 \\
0 & 0 & 0.7597 \\
0 & 1 & 0
\end{array}\right], F 3=J_{0-3}^{T} \times\left[\begin{array}{c}
0 \\
-0.5 \\
0 \\
0 \\
0 \\
0
\end{array}\right]=\left[\begin{array}{c}
0 \\
0.38 \\
0
\end{array}\right]
$$

\section{Discussions}

After analyzing and comparing joints' inputs from both robots to establish static balancing, motion analysis compares these two robots described in this section. Static balance refers to the ability of stationary on the object to its balance. This happens when the object's center of gravity is on the axis of rotation. Whereas dynamic balance is an object's ability to balance while in motion or when switching between positions First, the 3D model for these two robot types has designed using CATIA Version 5R21 software (Dassault Systems, Waltham, Massachusetts, USA) (Figures 2 and 3). These models had transferred to MSC Adams Version 2018 software (MSC Software Corp, Santa Ana, California, USA) for motion simulation. Computer simulation is a common method in biology field analysis ${ }^{30-39}$. Therefore, in this study, two robot types were compared using Euler-Lagrange, kinetic, and kinematic equations in MSC Adams software. Figure 4 shows that in the same condition, maximum velocity in the contact location of the end-effector with the tumor for robot type 1 is 1.7 times more than the maximum velocity for robot type 2 . This shows that robot type 1 is more effective than type 2 . Motion period in robot type 1 has two peaks, while robot type 2 has only one peak. This means that robot type 2 has a better kinematic range, but robot type 1 can move more delicately, especially during ablation.

The kinetic view shows that the maximum end-effector force of robot type 1 applied to the tumor is more than 1.8 times than in robot type 2 (Figure 5). This means that ablation is more comfortable in robot type 1 because, in the same conditions, robot type 1 can perform ablation $80 \%$ better than robot type 2 . On the other hand, the

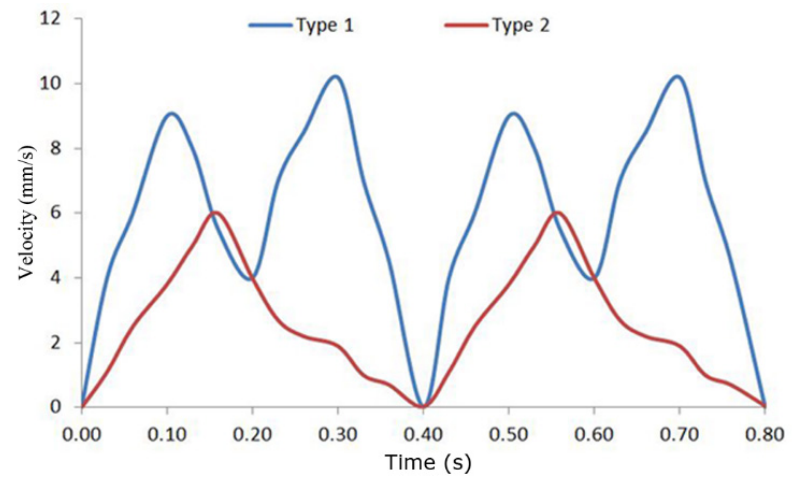

Figure 4. Comparison of velocity diagram for end-effectors of robot types 1 and 2 .

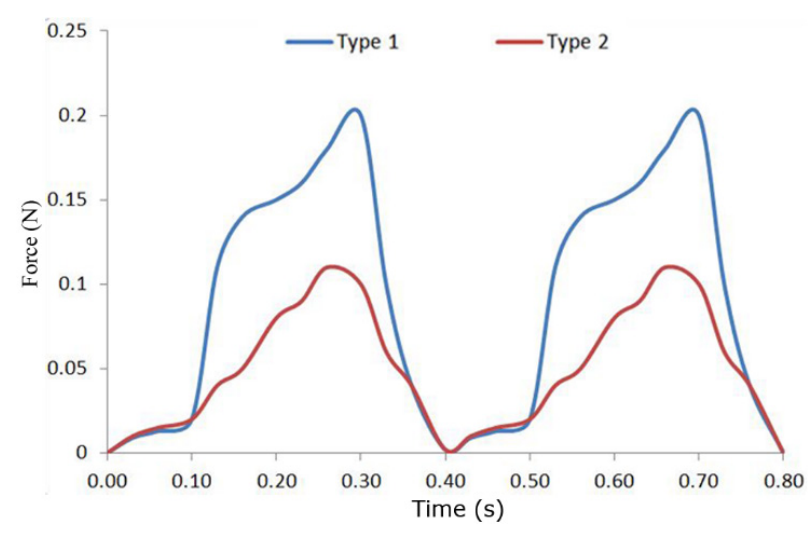

Figure 5. Comparison of force diagram for end-effectors of robot types 1 and 2 .

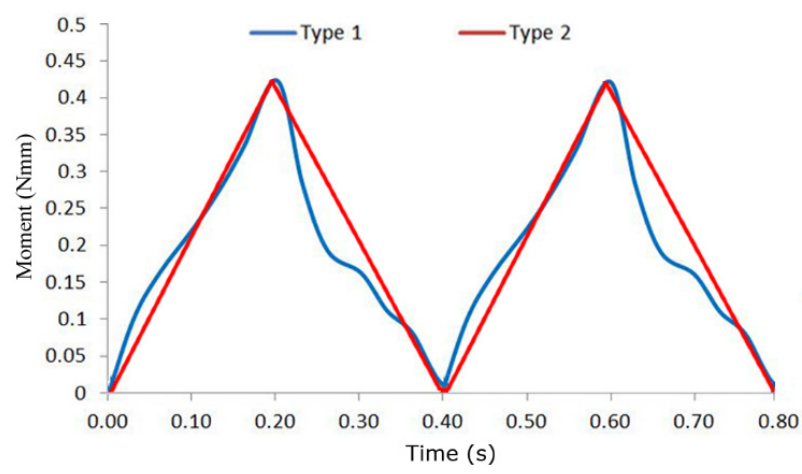

Figure 6. Comparison of moment diagram for end-effectors of robot types 1 and 2 .

maximum moment for both robot types is the same with less than a $1 \%$ difference (Figure 6). This means that the end-effector's output power during ablation and resection is the same for both types. Moment graph in robot type 2 is linear, while this graph is parabolic in robot type 1 . Linear moment graph in robot type 2 is a significant disadvantage in these robots during surgeries since it prevents smooth motion for the robot. Therefore, this issue turns out to be a significant weakness of robot type 2 . 


\section{Conclusion}

Robot type 1 and robot type 2 have almost the same endeffector output power for ablation and resection. On the other hand, robot type 2 has a more straightforward mechanism, fewer joints, and a better kinematic range than robot type 1 . However, the results of this study show that for ablation and resection of a brain tumor, it is better to use single port minimal invasive surgical robots type 1 . Because in the same conditions, the damaged volume of cerebral cortex tissue during resection is more for robot type 2, which results in post-surgery risks. Moreover, robot type 2 needs more internal loading in its joints for establishing static balancing. Robot type 1 has more smooth motions while it has better performance for ablation by $80 \%$.

\section{Conflict of Interest}

The authors declare that they have no conflict of interests

\section{Ethical Statement}

Not applicable

\section{References}

1. Beasley RA. Medical robots: current systems and research directions. Journal of Robotics J Robot. 2012;2012:401613. doi: 10.1155/2012/401613.

2. Gholampour S, Bahmani M, Shariati A. Comparing the efficiency of two treatment methods of hydrocephalus: shunt implantation and endoscopic third ventriculostomy. Basic Clin Neurosci. 2019;10(3):185-98. doi: 10.32598/bcn.9.10.285.

3. Liounakos Jl, Wang MY. Lumbar 3-Lumbar 5 RoboticAssisted Endoscopic Transforaminal Lumbar Interbody Fusion: 2-Dimensional Operative Video. Oper Neurosurg (Hagerstown). 2020;19(1):E73-E4. doi: 10.1093/ons/opz385.

4. Taher M, Gholampour S. Effect of ambient temperature changes on blood flow in anterior cerebral artery of patients with skull prosthesis. World Neurosurg. 2020;135:e358-e65. doi: 10.1016/j.wneu.2019.11.171.

5. Guo Z, Dong Z, Lee KH, Cheung CL, Fu HC, Ho JD, et al. Compact design of a hydraulic driving robot for intraoperative MRI-guided bilateral stereotactic neurosurgery. IEEE Robot Autom Lett. 2018;3(3):2515-22. doi: 10.1109/ Ira.2018.2814637.

6. Gholampour S. FSI simulation of CSF hydrodynamic changes in a large population of non-communicating hydrocephalus patients during treatment process with regard to their clinical symptoms. PLoS One. 2018;13(4):e0196216. doi: 10.1371/ journal.pone.0196216.

7. Gholampour S, Soleimani N, Zalii AR, Zare Karizi F, Seddighi A. Numerical simulation of the cervical spine in a normal subject and a patient with intervertebral cage under various loadings and in various positions. Int Clin Neurosci J. 2016;3(2):92-8. doi: 10.22037/icnj.v3i2.13170.

8. Trybula SJ, Oyon DE, Wolinsky JP. Robotic tissue manipulation and resection in spine surgery. Neurosurg Clin N Am. 2020;31(1):121-9. doi: 10.1016/j.nec.2019.08.014.

9. Gholampour S, Fatouraee N, Seddighi AS, Seddighi A. Evaluating the effect of hydrocephalus cause on the manner of changes in the effective parameters and clinical symptoms of the disease. J Clin Neurosci. 2017;35:50-5. doi: 10.1016/j. jocn.2016.09.012.

10. Naghibzadeh M, Gholampour S, Naghibzadeh M, SadeghianNodoushan F, Nikukar H. The effect of electromagnetic field on decreasing and increasing of the growth and proliferation rate of dermal fibroblast cell. Dermatol Ther. 2020:e13803. doi: $10.1111 /$ dth.13803.

11. SedaghatY, Gholampour S, Tabatabai Ghomshe F. Comparison of the effectiveness of manual cleaning, hydrogen peroxide vapour and ultraviolet-c in disinfection of hospital equipment. Infektološki Glasnik. 2019;39(3):66-84. doi: 10.37797/ ig.39.3.2.

12. Aprile I, Germanotta M, Cruciani A, Loreti S, Pecchioli C, Cecchi $F$, et al. Upper limb robotic rehabilitation after stroke: a multicenter, randomized clinical trial. J Neurol Phys Ther. 2020;44(1):3-14. doi: 10.1097/npt.0000000000000295.

13. Le XF, Shi Z, Wang QL, Xu YF, Zhao JW, Tian W. Rate and risk factors of superior facet joint violation during cortical bone trajectory screw placement: a comparison of robot-assisted approach with a conventional technique. Orthop Surg. 2020;12(1):133-40. doi: 10.1111/os.12598.

14. Gholampour S, Haghighi Hassanali Deh H. The effect of spatial distances between holes and time delays between bone drillings based on examination of heat accumulation and risk of bone thermal necrosis. Biomed Eng Online. 2019;18(1):65. doi: 10.1186/s12938-019-0686-6.

15. Haber GP, White MA, Autorino R, Escobar PF, Kroh MD, Chalikonda $S$, et al. Novel robotic da Vinci instruments for laparoendoscopic single-site surgery. Urology. 2010;76(6):1279-82. doi: 10.1016/j.urology.2010.06.070.

16. Hajirayat K, Gholampour S, Sharifi I, Bizari D. Biomechanical simulation to compare the blood hemodynamics and cerebral aneurysm rupture risk in patients with different aneurysm necks. J Appl Mech Tech Phys. 2017;58(6):968-74. doi: 10.1134/s0021894417060025.

17. Prideaux N, van den Berg M, Drummond C, Barr C. Augmented performance feedback during robotic gait therapy results in moderate intensity cardiovascular exercise in subacute stroke. J Stroke Cerebrovasc Dis. 2020;29(6):104758. doi: 10.1016/j. jstrokecerebrovasdis.2020.104758.

18. Gholampour S, Hajirayat K. Minimizing thermal damage to vascular nerves while drilling of calcified plaque. BMC Res Notes. 2019;12(1):338. doi: 10.1186/s13104-019-4381-2.

19. Sergeeva A, Huysman M, Faraj S. Transforming Work Practices of Operating Room Teams: The Case of the Da Vinci robot. ICIS; 2015.

20. Seung S, Liu P, Park S, Park J-O, Ko SY. Single-port robotic manipulator system for brain tumor removal surgery: SiromanS. Mechatronics. 2015;26:16-28. doi: 10.1016/j. mechatronics.2014.12.002.

21. Hu D, Jiang Y, Belykh E, Gong Y, Preul MC, Hannaford B, et al. Toward real-time tumor margin identification in image-guided robotic brain tumor resection. In: Webster lii RJ, Fei B, eds. Medical Imaging 2017: Image-Guided Procedures, Robotic Interventions, and Modeling. International Society for Optics and Photonics; 2017. doi: 10.1117/12.2255417.

22. Heredia S, Marinho MM, Ikemoto T, Harada K, Mitsuishi M, Padilla M, et al. Development of a virtual reality simulator for robotic brain tumor resection. 2016 International Symposium on Micro-NanoMechatronics and Human Science (MHS). Nagoya, Japan: IEEE; 2016. doi: 10.1109/mhs.2016.7824185.

23. Hu D, Gong Y, Hannaford B, Seibel EJ. Semi-autonomous simulated brain tumor ablation with ravenii surgical 
robot using behavior tree. IEEE Int Conf Robot Autom. 2015;2015:3868-75. doi: 10.1109/icra.2015.7139738.

24. Chinbe H, Yoneyama T, Watanabe T, Miyashita K, Nakada $M$. Finger-attachment device for the feedback of gripping and pulling force in a manipulating system for brain tumor resection. Int J Comput Assist Radiol Surg. 2018;13(1):3-12. doi: 10.1007/s11548-017-1640-3.

25. Azarnoush H, Siar S, Sawaya R, Zhrani GA, WinklerSchwartz A, Alotaibi FE, et al. The force pyramid: a spatial analysis of force application during virtual reality brain tumor resection. J Neurosurg. 2017;127(1):171-81. doi: 10.3171/2016.7.jns16322.

26. Niccolini M, Castelli V, Diversi C, Kang B, Mussa F, Sinibaldi E. Development and preliminary assessment of a robotic platform for neuroendoscopy based on a lightweight robot. The International Journal of Medical Robotics and Computer Assisted Surgery Int J Med Robot. 2016;12(1):4-17. doi: 10.1002/rcs.1638.

27. Lefranc M, Capel C, Pruvot-Occean AS, Fichten A, Desenclos C, Toussaint $\mathrm{P}$, et al. Frameless robotic stereotactic biopsies: a consecutive series of 100 cases. J Neurosurg. 2015;122(2):34252. doi: 10.3171/2014.9.jns14107.

28. Maddahi Y, Gan LS, Zareinia K, Lama S, Sepehri N, Sutherland GR. Quantifying workspace and forces of surgical dissection during robot-assisted neurosurgery. Int J Med Robot. 2016;12(3):528-37. doi: 10.1002/rcs.1679.

29. Ross WA, Hill WM, Hoang KB, Laarakker AS, Mann $\mathrm{BP}$, Codd PJ. Automating neurosurgical tumor resection surgery: Volumetric laser ablation of cadaveric porcine brain with integrated surface mapping. Lasers Surg Med. 2018;50(10):1017-24. doi: 10.1002/lsm.23000.

30. Gholampour S, Gholampour H, Khanmohammadi H. Finite element analysis of occlusal splint therapy in patients with bruxism. BMC Oral Health. 2019;19(1):205. doi: 10.1186/ s12903-019-0897-z.

31. Gholampour S, Jalali A. Thermal analysis of the dentine tubule under hot and cold stimuli using fluid-structure interaction simulation. Biomech Model Mechanobiol. 2018;17(6):1599610. doi: 10.1007/s10237-018-1046-3.

32. Gholampour s GH. Correlation of a new hydrodynamic index with other effective indexes in Chiari I malformation patients with different associations. Scientific Reports. 2020.

33. Gholampour S, Mehrjoo S. Effect of bifurcation in the hemodynamic changes and rupture risk of small intracranial aneurysm. Neurosurg Rev. 2020. doi: 10.1007/s10143-02001367-3.

34. Haberland M, Kim S. On extracting design principles from biology: II. Case study-the effect of knee direction on bipedal robot running efficiency. Bioinspir Biomim. 2015;10(1):016011. doi: 10.1088/1748-3190/10/1/016011.

35. Hinkel G, Groenda H, Krach S, Vannucci L, Denninger $\mathrm{O}$, Cauli N, et al. A framework for coupled simulations of robots and spiking neuronal networks. J Intell Robot Syst. 2017;85(1):71-91. doi: 10.1007/s10846-016-0412-6.

36. Kelty CM. Robot life: simulation and participation in the study of evolution and social behavior. Hist Philos Life Sci. 2018;40(1):16. doi: 10.1007/s40656-017-0181-y.

37. Liu Y, Sun S, Wu X, Mei T. A wheeled wall-climbing robot with bio-inspired spine mechanisms. J Bionic Eng. 2015;12(1):1728. doi: 10.1016/S1672-6529(14)60096-2.

38. Porez M, Boyer F, ljspeert AJ. Improved Lighthill fish swimming model for bio-inspired robots: modeling, computational aspects and experimental comparisons. Int J Rob Res. 2014;33(10):1322-41. doi: 10.1177/0278364914525811.

39. Zhang J, Wang T, Wang J, Li B, Hong J, Zhang JXJ, et al. Dynamic modeling and simulation of inchworm movement towards bio-inspired soft robot design. Bioinspir Biomim. 2019;14(6):066012. doi: 10.1088/1748-3190/ab3e1f. 